\title{
Spectroscopic Studies on Distorted Structure Nanomolecules by Using Lie Algebraic Model
}

\author{
S. Rao Karumuri ${ }^{a, *}$, K. Girija Sravani ${ }^{b}$, J. VijayshekaR ${ }^{c}$ And L.S.S. Reddy ${ }^{d}$ \\ ${ }^{a}$ Department of Electronics and Instrumentation, Lakireddy Bali Reddy College of Engineering \\ Mylavaram, Andhra Pradesh, India \\ ${ }^{b}$ Department of Electronics \& Communication, Lingaya's Institute of Management \& Technology \\ Andhra Pradesh, India \\ ${ }^{c}$ Department of Mathematics, GITAM University, Hyderabad, Andhra Pradesh, India \\ ${ }^{d}$ Department of Computer Science \& Engineering, Lakireddy Bali Reddy College of Engineering \\ Mylavaram, Andhra Pradesh, India
}

(Received September 20, 2011; in final form April 24, 2012)

\begin{abstract}
We have applied Lie algebraic model to distorted structure molecules to determine the vibrational spectra of different stretching and bending vibrational modes. The the Lie algebraic model of the Hamiltonian expression is $H=E_{0}+\sum_{i=1}^{n} A_{i} C_{i}+\sum_{i<j}^{n} A_{i j} C_{i j}+\sum_{i<j}^{n} \lambda_{i j} M_{i j}$. By using the Lie algebraic method, the stretching vibrational energies of fullerene $\left(C_{80}\right)$ are calculated in the one-dimensional [U(2)] framework. Using the model Hamiltonian so constructed, we have calculated the local mode vibrational energy levels of the fullerene $\left(C_{80}\right)$ accurately.
\end{abstract}

PACS: 03.65.Fd, 07.57.-c, 02.20.Sv, 78.30.Na

\section{Introduction}

Molecular spectroscopy is an area of active interest from many standpoints. Due to its numerous connections with other scientific areas, this branch of modern physics is playing an essential role in both experimental and theoretical approaches [1] to understanding a huge number of important problems. In recent years, molecular spectroscopy [2] has been going through an exciting time of renewed interest [3-7] which, once again, is being fueled by the rapid development of sophisticated experimental approaches. The algebraic methods have been found to be very much useful in the study of vibrational spectra of medium and large molecules using the Lie algebra, especially after the development of quantum mechanics in the first part of 20th century. The interesting area of current research in molecular physics is to interpret the experimental data with the help of theoretical models. Two traditional approaches like Dunham-like expansion of energy levels in terms of rotation-vibration quantum number and the solution of the Schrödinger equation with potentials have been used so far in the analysis of experimental data. The new theoretical concept — vibron model (based on the Lie algebra) to study molecular spectra was built in the last part of the 20 th century. This new model seems to offer a concrete and complementary technique to the traditional approaches used in molecular

* corresponding author; e-mail: srinivasakarmuri@gmail.com spectroscopy. The algebraic model (vibron model) originally developed for diatomic and tri-atomic molecules. $\mathrm{U}(4)$ and $\mathrm{U}(2)$ algebraic model been used so far in the analysis of experimental data [8]. The Lie algebraic approach has been successfully applied to the study of the vibrational frequencies of $\mathrm{HCN}, \mathrm{HCCF}, \mathrm{HCCD}$, tetrahedral and nickel metalloporphirins. The stretching vibrations of some polyatomic molecules like n-alkane molecule and polyethylene have been described using $\mathrm{U}(2)$ algebra.

In order to deal with these problems, a local mode method contrasted with the usual normal mode analysis has been developed. The theoretical description of the observed features has so far mostly focused on the study of the stretching modes but not the bending modes. Thus, the theoretical studies of excited vibrational states of molecules provide new challenges.

In this paper, we have applied the algebraic methods to the study of the vibrational energy levels of distorted structure of nanomolecules and compare results with experimental values.

\section{Approach to algebraic method with Lie algebra}

In the last few years, an algebraic method has been introduced as a computational tool for the analysis and interpretation of experimental ro-vibrational spectra of small- and medium-size molecules. This method has been used extensively in chemical physics and molecular physics. This method is based on the idea of dynamic symmetry, which, in turn, is expressed through 
the language of the Lie algebras. By applying algebraic techniques [3-5], one obtains an effective Hamiltonian operator that conveniently describes the ro-vibrational degrees of freedom of the physical system. Within this framework, any specific mechanism relevant to the correct characterization of the molecular dynamics and spectroscopy can be accounted for. The algebraic methods [9] are formulated in such a way that they contain the same physical information of both $a b$ initio theories (based on the solution of the Schrödinger equation) and of semi-empirical approaches (making use of phenomenological expansions in powers of appropriate quantum numbers). However, by employing the powerful method of group theory [10], the results can be obtained in a more rapid and straightforward way. Now this approach and its hybrid is used to treat polyatomic vibrations and various other problems.

In recent years, algebraic models, such as the Lie algebraic methods and boson-realization model have been proposed for the descriptions of vibrations, rotations $[11,12]$, and rotation-vibration interactions in polyatomic molecules. Algebraic methods have been extensively used to study problems in nuclear physics, molecular physics and quantum optics etc., for example, the problems of vibrational excited states and potential energy surfaces for small polyatomic molecules have been solved successfully [13-15]. The U(4) model deals with the rotation and the vibration simultaneously, but it becomes quite complicated when the number of atoms in a molecule are more than four. The U(2) model [8] was particularly successful in explaining stretching vibrations of polyatomic molecules such as benzene-like and octahedral molecules. This model was extended to deal with stretching vibrations in triatomic molecules. In this paper, we use the $\mathrm{U}(2)$ algebraic model to study the higher excited vibrations of fullerenes such as $C_{60}, C_{70}, C_{74}, C_{80}$ and other molecules. In this paper we concentrate only on the study of $C_{80}$ molecule.

The $C_{80}$ has been termed as one of the three "missing fullerenes" due to its extremely low abundance in the Huffman-Kratschmer soot. Recently, however, two $C_{80}$ isomers were synthesized by Dennis and Shinohara, as a byproduct of the latter's work in generating endohedral metallofullerenes [14]. One of these was first noted as a very-late-eluting fraction in HPLC separations using buckyprep columns; analysis with CNMR established its $D_{5 d}$ symmetry (Fig. 1) [15].

It was felt that this species would be an ideal subject for photo-physical analysis. Its strong retention on HPLC columns helps to keep it free from contamination by fullerenes of different mass, and by isomers of different structure. Moreover, its structure is directly related to $C_{60}$ and $C_{70}$ via an extension of the famous ten-carbon-addition sequence: $\mathrm{C}_{60}\left\{I_{h}\right\} \rightarrow \mathrm{C}_{70}\left\{D_{5 d}\right\} \rightarrow \mathrm{C}_{80}\left\{D_{5 d}\right\}$. Since the two lower species have high $\Phi_{\text {ISC }}$ values, strong transient absorptivities, and long triplet lifetimes, it was through that $C_{80}\left\{D_{5 d}\right\}$ might display analogous properties [16].

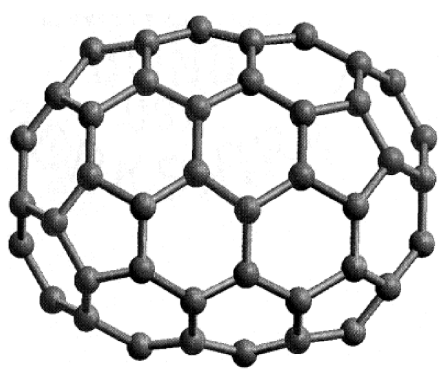

Fig. 1. $C_{80}\left\{D_{5 d}\right\}$.

The $C_{80}\left\{D_{5 d}\right\}$ isomer lacks appreciable transient absorption [17], this is apparently due to a combination of negligible quantum yield and small triplet-state epsilons. It does quench singlet oxygen emission, albeit at about $1 / 3$ rd of the diffusion limit this suggests that the $T_{1}$ state of $C_{80}\left\{D_{5 d}\right\}$ is within $1 \mathrm{kT}\left(\approx 300 \mathrm{~cm}^{-1}\right)$ of $7880 \mathrm{~cm}^{-1}$.

In constructing this $\mathrm{U}(2)$ algebraic model, we use the isomorphism of the Lie algebra of $\mathrm{U}(2)$ with that of the one-dimensional Morse oscillator. The eigenstates of the one-dimensional Schrödinger equation, with a Morse potential

$$
h(p x)=p_{2} / 2 \mu+D[1-\exp (-\alpha x)]^{2}
$$

can be put into one to one correspondence with the representations of $\mathrm{U}(2) \supset O(2)$, characterized by the quantum numbers $|N, m\rangle$ with the provision that one takes only the positive branch of $m$, i.e. $m=N, N-2, \ldots, 1$ or 0 for $N=$ odd or even $(N=$ integer $)$. The Morse Hamiltonian (1) corresponds in the $\mathrm{U}(2)$ basis to a simple Hamiltonian, $h=\bar{C}_{0}+A C$, where $C$ is the invariant operator of $\mathrm{O}(2)$, with eigenvalues $\left(m^{2}-N^{2}\right)$.

The eigenvalues of $h$ are

$$
\begin{aligned}
& \bar{C}=\bar{C}_{0}+A\left(m^{2}-N^{2}\right), \\
& m=N, N-2, \ldots 1 \text { or } 0 \quad(N=\text { integer }) .
\end{aligned}
$$

Introducing the vibrational quantum number $\nu=(N-$ $m) / 2$, Eq. (2) can be rewritten as

$$
\begin{aligned}
& \bar{C}=\bar{C}_{0}-4 A\left(N \nu-\nu^{2}\right), \quad \nu=0,1, \ldots N / 2 \\
& \quad \text { or } \quad N-1 / 2(N=\text { even or odd }) .
\end{aligned}
$$

The value of $C_{0}, A$, and $N$ are given in terms of $\mu, D$ and $\alpha$ by $C_{0}=-D,-4 A N=h \alpha(2 D / \mu)^{1 / 2}, 4 A=$ $-h^{2} \alpha^{2} / 2 \mu$. One can immediately verify that these are the eigenvalues of the Morse oscillator.

Now consider a molecule with $n$ bonds. In the algebraic model, each bond $i$ is replaced by an algebra (here $\mathrm{U}_{i}(2)$ ), with Hamiltonian $h_{i}=\bar{C}_{0 i}+A_{i} C_{i}$ where $C_{i}$ is the invariant operator of $\mathrm{O}_{i}(2)$ with eigenvalues $-4\left(N_{i} \nu_{i}-\nu_{i}^{2}\right)$. The bonds interact with a bond-bond interaction. Two types of interaction are usually considered, which we denote by $C_{i j}$ and $M_{i j}$ and are called the Casimir and Majorana [8] interactions, respectively.

The algebraic model Hamiltonian [18] we consider is thus 


$$
H=E_{0}+\sum_{i=1}^{n} A_{i} C_{i}+\sum_{i<j}^{n} A_{i j} C_{i j}+\sum_{i<j}^{n} \lambda_{i j} M_{i j} .
$$

In Eq. (4) $C_{i}$ is an invariant operator with eigenvalues $4\left(\nu_{i}^{2}-N_{i} \nu_{i}\right)$ and the operator $C_{i j}$ is diagonal with matrix elements

$$
\begin{aligned}
& \left\langle N_{i}, \nu_{i} ; N_{j}, \nu_{j}\left|C_{i j}\right| N_{i}, \nu_{i} ; N_{j}, \nu_{j}\right\rangle \\
& \quad=4\left[\left(\nu_{i}+\nu_{j}\right)^{2}-\left(\nu_{i}+\nu_{j}\right)\left(N_{i}+N_{j}\right)\right],
\end{aligned}
$$

while the operator $M_{i j}$ has both diagonal and non-diagonal matrix element,

$$
\begin{aligned}
& \left\langle N_{i}, \nu_{i} ; N_{j}, \nu_{j}\left|M_{i j}\right| N_{i}, \nu_{i} ; N_{j}, \nu_{j}\right\rangle \\
& \quad=\left\langle N_{i} \nu_{j}+N_{j} \nu_{i}-2 \nu_{i} \nu_{j}\right\rangle, \\
& \quad\left\langle N_{i}, \nu_{i}+1 ; N_{j}, \nu_{j}-1\left|M_{i j}\right| N_{i}, \nu_{i} ; N_{j}, \nu_{j}\right\rangle \\
& \quad=-\left[\nu_{j}\left(\nu_{i}+1\right)\left(N_{i}-\nu_{i}\right)\left(N_{j}-\nu_{j}+1\right)\right]^{1 / 2}, \\
& \left\langle N_{i}, \nu_{i}-1 ; N_{j}+1\left|M_{i j}\right| N_{i}, \nu_{i} ; N_{j}, \nu_{j}\right\rangle \\
& \quad=-\left[\nu_{i}\left(\nu_{j}+1\right)\left(N j-\nu_{j}\right)\left(N_{i}-\nu_{i}+1\right)\right]^{1 / 2} .
\end{aligned}
$$

Equation (6) is a generalization of the two-bond model to $n$ bonds.

The simplest basis to diagonalize the Hamiltonian is characterized by the representation of local mode chain, where below each group we have used quantum numbers characterizing the eigenvalues of the corresponding invariant operator. $N$ is the number of bosons related to stretching physical modes. The quantum numbers $v_{i}$ correspond to the number of quanta in each oscillator while $V$ is the total vibrational quantum number given by $[3-5]$,

$$
V=\sum_{i=1}^{n} v_{i}
$$

For a particular polyad, the total vibrational quantum number is always conserved. The inclusion of $M_{i j}$ in the local Hamiltonian operator cannot affect the conservation rule. In Eq. (5), $C_{i}$ is an invariant operator of uncoupled bond with eigenvalues $4\left(\nu_{i}^{2}-N_{i} \nu_{i}\right)$ and the operator $C_{i j}$ for coupled bonds are diagonal with matrix elements $[3,4]$.

\section{Results and discussions}

We have used $\mathrm{U}(2)$ algebraic model to study the vibrational spectra of the fullerene $\left(C_{80}\right.$ and $\left.C_{80}-\mathrm{O}\right)$ molecules with fewer algebraic parameters (i.e. $A, A^{\prime}, \lambda$ and $N$ (vibron number)).

The values of vibron number $(N)$ can be determined by the relation

$$
N_{i}=\frac{\omega_{e}}{\omega_{e} \chi_{e}}-1, \quad(i=1,2, \ldots),
$$

where $\omega_{e}$ and $\omega_{e} \chi_{e}$ are the spectroscopic constants of diatomic molecules of stretching interaction of the molecule considered. This numerical value must be seen as initial guess; depending on the specific molecular structure, one can expect changes in such an estimate, which, however, should not be larger than $\pm 20 \%$ of the original value [Eq. (8)]. The vibron number $N$ between the diatomic molecules C-C are 140. From Fig. 1, it is noticed that some of the bonds are equivalent. It may be noted that during the calculation of the vibrational frequencies of fullerene $C_{80}$ and $C_{80}-\mathrm{O}$, the value of $N$ is kept fixed and not used as free parameter.

The second step is to obtain a starting guess for the parameter $A$. As such, the expression for the single-oscillator fundamental mode as

$$
E(\nu-1)=-4 A(N-1) .
$$

In the present case we have different energies, corresponding to symmetric and antisymmetric combinations of the different local modes. A possible strategy is to use the center of gravity of these modes, so the guess for

$$
\bar{A}=\frac{\bar{E}}{4(1-N)} .
$$

The third step is to obtain an initial guess for $\lambda$. Its role is to split the initially degenerate local modes, placed here at the common value $E$ used in Eq. (9). Such an estimate is obtained by considering the simple matrix structure, we can find

$$
\lambda=\frac{\left|E_{1}-E_{2}\right|}{2 N} .
$$

A numerical fitting procedure to adjust (in a least-squares sense, for example) the parameters $A$ and $\lambda$ starting from values Eq. (10) and Eq. (11), and $A^{\prime}$ (whose initial guess can be zero).

To get an estimation of the precession of both observed and calculated data, we use standard deviation (SD) denoted by $\sigma$ at the bottom of two tables

$$
\sigma^{2}=\frac{1}{o-p} \sum_{i=1}^{o}\left(E_{\mathrm{cal}}^{i}-E_{\mathrm{obs}}^{i}\right)^{2}
$$

where $o$ is the number of observed data and $p$ is the number of parameters used in the fitting.

The fitting parameters used in the study of vibrational spectra of buckminsterfullerene $\left(C_{80}\right)$ is given in Table I.

TABLE I

Fitting parameters used in the study of fullerene $\left(C_{80}\right)$.

\begin{tabular}{c|c|c|c}
\hline \hline Vibron number & \multicolumn{3}{|c}{ Stretching algebraic parameters $\left[\mathrm{cm}^{-1}\right]$} \\
\hline$N$ & $A$ & $\lambda$ & $A^{\prime}$ \\
\hline 140 & -0.7463 & 0.1562 & -0.0562
\end{tabular}

\section{Conclusion}

Using model Hamiltonian, we have presented an algebraic model of coupled one-dimensional Morse oscillators which can be used to describe $\mathrm{C}-\mathrm{C}$ stretching vibrations of polyatomic molecules with good accuracy. The model presented here describes the splitting of local $\mathrm{C}-\mathrm{C}$ stretching modes due to residual inter-bond interactions. The splitting pattern determines the nature of interaction. Once we get the parameter, one then predicts the splitting pattern of overtones. It is worth pointing out that most applications of the previous algebraic models are restricted to vibrations of gas molecules. 
TABLE II

Simulated and calculated energies $\left(\mathrm{cm}^{-1}\right)$ of fullerene $\left(C_{80}\right)[19]$ for $V=1$.

\begin{tabular}{c|c|c|c}
\hline \hline Normal level & \multicolumn{2}{|c|}{ Fullerene $\left(C_{80}\right)$ molecule } & Percentage of deviation \\
\cline { 2 - 3 } & I Ref. [19] & II This study & $\Delta|I-I| / I \times 100[\%]$ \\
\hline$\nu_{1}$ & 162.92 & 162.78 & $0.023 \%$ \\
$\nu_{2}$ & 227.54 & 228.38 & $0.197 \%$ \\
$\nu_{3}$ & 306.89 & 307.46 & $0.343 \%$ \\
$\nu_{4}$ & 376.13 & 375.23 & $0.238 \%$ \\
$\nu_{5}$ & 442.04 & 442.89 & $0.031 \%$ \\
$\nu_{6}$ & 470.79 & 472.29 & $0.456 \%$ \\
$\nu_{7}$ & 492.65 & 490.33 & $0.193 \%$ \\
$\nu_{8}$ & 515.37 & 514.39 & $0.147 \%$ \\
$\nu_{9}$ & 540.74 & 540.09 & $0.019 \%$ \\
$\nu_{10}$ & 607.34 & 606.78 & $0.145 \%$ \\
$\nu_{11}$ & 638.44 & 637.89 & $0.148 \%$ \\
$\nu_{12}$ & 655.08 & 654.09 & $0.146 \%$ \\
$\nu_{13}$ & 684.37 & 681.56 & $0.198 \%$ \\
$\nu_{14}$ & 704.19 & 704.87 & $0.018 \%$ \\
$\nu_{15}$ & 733.64 & 734.88 & $0.113 \%$ \\
$\nu_{16}$ & 768.40 & 767.77 & $0.145 \%$ \\
$\nu_{17}$ & 787.92 & 790.64 & $0.216 \%$ \\
$\nu_{18}$ & 833.73 & 836.56 & $0.254 \%$ \\
$\nu_{19}$ & 857.38 & 860.34 & $0.331 \%$ \\
$\nu_{20}$ & 868.71 & 869.33 & $0.116 \%$ \\
$\nu_{21}$ & 901.56 & 899.38 & $0.142 \%$ \\
$\nu_{22}$ & 912.58 & 910.99 & $0.196 \%$ \\
$\nu_{23}$ & 957.21 & 954.66 & $0.199 \%$ \\
$\nu_{24}$ & 1066.85 & 1063.52 & $0.198 \%$ \\
$\nu_{25}$ & 1183.11 & 1179.99 & $0.172 \%$ \\
$\nu_{26}$ & 1214.91 & 1215.62 & $0.001 \%$ \\
\hline$\Delta(\mathrm{RMS})=2.381 \mathrm{~cm}-1$ & & \\
& & & \\
\hline
\end{tabular}

In this study, vibrational spectra of fullerene for 26 vibrational bands, we obtain the RMS deviation i.e. $\Delta(\mathrm{RMS})=2.381 \mathrm{~cm}^{-1}$ from Table II. The present calculations demonstrate that $\mathrm{U}(2)$ model can be applied successfully to the molecules having 80 number of atoms or even more than that. More accurate results can be obtained if the interaction between the stretch and bend is considered. It is believed that once we get the observed energy levels of higher overtones and combination bands, the comparison of calculated bands can be easily done with the help of model Hamiltonian using the parameters.

Hence, the results of our calculation on $C_{80}$ considering its both structural and vibrational properties by algebraic method and comparing the results obtained by semi-empirical PM3 [20] calculations, establish the nearest precision.

We hope that this work will stimulate further research in analysis of vibrational spectra of other nanomolecules like protein molecules where the algebraic approach has not been applied so far.

\section{Acknowledgments}

The author Srinivasa Rao Karumuri would like to thank Prof. Thomson G. Spiro for providing necessary literature for this study.
The author Srinivasa Rao Karumuri also would like to thank the Department of Science and Technology, New Delhi, India, for providing the financial assistance for this study. The author is very thankful to the anonymous referee of this paper for valuable suggestions and comments, which greatly helped to improve the quality of this paper.

\section{References}

[1] A. Arima, F. Iachello, Phys. Rev. Lett. 35, 1069 (1975).

[2] F. Iachello, Chem. Phys. Lett. 78, 581 (1981).

[3] F. Iachello, S. Oss, Phys. Rev. Lett. 66, 2976 (1991).

[4] F. Iachello, S. Oss, J. Mol. Spectrosc. 153, 225 (1992).

[5] F. Iachello, S. Oss, Chem. Phys. Lett. 205, 285 (1993).

[6] F. Iachello, S. Oss, J. Chem. Phys. 99, 7337 (1993).

[7] F. Iachello, S. Oss, J. Chem. Phys. 104, 6956 (1996).

[8] F. Iachello, S. Oss, Eur. Phys. J. D 19, 307 (2002).

[9] F. Iachello, R.D. Levine, J. Chem. Phys. 77, 3046 (1982).

[10] Y. Alhassid, F. Gursey, F. Iachello, Ann. Phys. (N.Y.) 148, 346 (1983).

[11] N.K. Sarkar, J. Choudhury, R. Bhattacharjee, Mol. Phys. 104, 3051 (2006).

[12] N.K. Sarkar, J. Choudhury, R. Bhattacharjee, Indian J. Phys. 82, 767 (2008).

[13] F. Iachello, Chem. Phys. Lett. 78, 581 (1981); O.S. van Roosmalen, I. Benjamin, R.D Levine, J. Chem. Phys. 81, 5986 (1984); O.S. van Roosmalen, I. Benjamin, R.D Levine, J. Chem. Phys. 79, 2515 (1983); Y. Zheng, S. Ding, Chem. Phys. 247, 225 (1999); Sci. China 44, 984 (2001); Int. J. Quant. Chem. 108, 1059 (2008).

[14] M. Wang, S. Ding, D. Feng, H. Liu, Phys. Rev. A 66, 022506-1 (2002).

[15] J.L. Ping, J.Q. Chen, Ann. Phys. (N.Y.) 255, 75 (1997).

[16] T. Muller, P. Vaccaro, F. Perez-Bernal, F. Iachello, J. Chem. Phys. 111, 5038 (1999).

[17] R. Lemus, A. Frank, J. Chem. Phys. 101, 8321 (1994).

[18] J. Choudhury, S.R. Karumuri, N.K. Sarkar, R. Bhattacharjee, Pramana J. Phys. 71, 439 (2008).

[19] M. Ibrahim, Acta Chim. Slov. 52, 153 (2005).

[20] M. Ibrahim, H. El-Haes, Chin. J. Phys. 43, 915 (2005). 